Relations industrielles

Industrial Relations

\title{
Ronald Sirard. La rédaction du contrat individuel de travail: pourquoi et comment
}

\section{Claude D'Aoust}

Volume 47, numéro 3, 1992

URI : https://id.erudit.org/iderudit/050802ar

DOI : https://doi.org/10.7202/050802ar

Aller au sommaire du numéro

Éditeur(s)

Département des relations industrielles de l'Université Laval

ISSN

0034-379X (imprimé)

1703-8138 (numérique)

Découvrir la revue

Citer ce compte rendu

D'Aoust, C. (1992). Compte rendu de [Ronald Sirard. La rédaction du contrat individuel de travail: pourquoi et comment]. Relations industrielles / Industrial Relations, 47(3), 585-587. https://doi.org/10.7202/050802ar

Tous droits réservés @ C Département des relations industrielles de l'Université Laval, 1992
Ce document est protégé par la loi sur le droit d'auteur. L’utilisation des services d'Érudit (y compris la reproduction) est assujettie à sa politique d'utilisation que vous pouvez consulter en ligne.

https://apropos.erudit.org/fr/usagers/politique-dutilisation/ 
labour education as playing a role in stemming this decline and as an instrument for attracting new members. A number of the contributors see new approaches to labour education as an important element in rejuvenating a divided and dispirited American society. volume.

An excellent bibliographical essay on worker education concludes the

At least two weaknesses can be noted in this collection of articles. The first is the disproportionate number of articles by authors whose activities are centered in the Northeastern part of the United States and who write from the perspective of that region. The second is the paucity of international comparisons of United States experience in labour education with that of other developed industrial countries. There is a single brief article on worker education in Western Europe. The absence of material on labour education in Canada is unfortunate. Given the historical ties between the Canadian and the United States labour movements, the high degree of economic integration of the neighboring countries, and the striking difference between the relatively stable Canadian union density rate and the declining United States rate, the book would have benefited from a study of the broad network of labour education programs in Canada with its summit in the twenty-five year old Labour College of Canada.

Sidney H. INGERMAN

McGill University

La rédaction du contrat individuel de travail: pourquol et comment, par Ronald Sirard avec la participation de Claire Armstrong, Montréal, Wilson \& Lafleur Ltée, 1990, 126 p., ISBN 2-89127-179-3

Ce volume se divise en deux parties, comme son titre le laisse entrevoir.

En premier lieu, les auteurs veulent nous expliquer "pourquoi» rédiger un contrat individuel de travail. Ce contrat étant consensuel, on peut se demander quelle est l'utilité d'un écrit qui le constate.

Il y a deux raisons qui militent en faveur de la rédaction d'un écrit. La première raison est que l'écrit valablement fait, c'est-à-dire dont la formation ou la validité ne sont pas contestées, ne peut être contredit par témoignage ou autrement. L'écrit constitue donc le moyen de preuve par excellence, en cas de litige futur.

L'autre raison a trait à la nécessité de préciser les droits et obligations de chaque partie. Ainsi, l'obligation de fournir une prestation de travail convenable étant un élément essentiel du contrat, il est inutile de le mentionner, sauf précisément lorsque l'employeur s'attend à un rendement exceptionnel. En cas d'exécution déficiente, l'employeur pourra, grâce à l'écrit, se départir plus facilement des services du salarié car ce document lui fournira une défense utile à la contestation d'un renvoi sous forme de poursuite en dommages et intérêts ou de réclamation de salaire d'échéance en échéance. En d'autres mots, il lui sera plus aisé de faire la preuve requise pour la résolution judiciaire de contrat préalablement résilié unilatéralement: le noeud du litige, en pareil cas, est 
l'intensité de l'obligation de fournir la prestation convenue. Corollairement, la même règle vaut lorsque le litige porte sur la nature du contrat: contrat d'entreprise ou contrat de travail? mandat salarié? etc. toutes distinctions qui ne se dégageront pas facilement, en certaines circonstances, de la subordination juridique, critère distinctif du contrat de travail qu'il n'est pas toujours aisé d'isoler.

L'autre motif pour coucher le contrat par écrit est de spécifier les clauses non essentielles au contrat, qui autrement seraient laissées à l'appréciation subjective du tribunal se fondant sur l'article 1024 C.C.B.-C.

Accessoirement, d'autres motifs justifient l'écrit. Ainsi en est-il des obligations nées d'une certaine expérience contractuelle ayant fait l'objet de délimitation jurisprudentielle. Par exemple, l'obligation de loyauté n'ayant pas suffi à résoudre tous les cas, les parties ont pris l'habitude, lorsque cela importe - mais aussi à titre de clause de style dans des situations qui ne l'exigeaient pas - de rédiger des clauses de non-concurrence. Celles-ci ont donné lieu à une importante moisson de jurisprudence, codifiée dans le nouveau Code civil1. Une partie peut avoir des raisons valables de rédiger une clause qui s'écarte, dans la mesure de la légalité, de ces règles générales.

Ces remarques et propositions se retrouvent dans la partie I de l'ouvrage. Mais celui-ci étant construit à partir de la doctrine et de la jurisprudence, l'analyse et la synthèse qui découle la clarté de la doctrine française font quelque peu défaut. Pour les tenants de la méthode empirique ou du raisonnement inductif, ou de la méthode de cas, cette caractéristique apparaîtra plutôt comme une qualité.

Pour ma part, cette dernière n'est pas la méthode que je favorise. Sa faiblesse ressort particulièrement des comparaisons ou analogies entre le droit civil et la common law. La discussion sur la soi-disant obligation de fiducie (fiduciary duty) (pp. 30 et seq.) ${ }^{2}$

La deuxième partie portant sur le "comment" rédiger est mieux réussie. On y trouve d'utiles conseils, par exemple celui de ne pas changer les termes pour le plaisir de varier, règle contraire à celle qui régit la rédaction littéraire. En droit, changer un mot, en effet, indique un changement de sens que les parties n'ont pas nécessairement voulu. Cela peut entraîner une catastrophe dans un litige.

De même faut-il éviter de surcharger le texte de mots inutiles et redondants dont le meilleur exemple est la fameuse expression "conjointement et solidairement", chère à nos confrères notaires, alors qu'il y a contradiction entre

1 L.Q. 1991 , c. 64, art. 2089.

2 Les juristes des provinces de common law se sont entichés de cette notion, sans s'attarder à ses origines ni donc à son utilité. En Angleterre, on parle d'obligation de fidelity ou de loyalty du salarié, comme on le fait traditionnellement au Québec.

Sur la prolifération de la notion de fiduciary duty au Canada (et son étonnement à ce sujet), voir: Sir Robert MEGARRY, "Historical Development", in The Law Society of Upper Canada (editor), Fiduciary Duties, Special Lectures 1990, Scarborough (Ont.), Thomson Professional Publishing Canada, 1991, à la p. 10. Voir aussi le texte de Peter D. MADDAUGH, p. 15 et seq. où, rapportant les propos d'un autre auteur, il qualifie de terme fiduciary de "peripatetic adjective». 
ces termes3. Un autre exemple est la manie d'utiliser «pour un dollar et autres considérations» qui est inspiré directement de la common law selon laquelle la considération (le fameux pepper grain du traité de droit contractuel anglais) fonde l'existence du contrat, par opposition à la rencontre des volontés qui préside à la formation des contrats en droit civil.

La partie 2 est, à mon avis, mieux réussie que la première non pas qu'elle soit plus originale mais en ce qu'elle ne fait pas (ou fait moins) double emploi avec la doctrine récente publiée sur le contrat individuel de travail en Québec.

Ce volume sera utile aux praticiens, et je dirais même aux étudiants universitaires qui suivraient un cours élaboré sur le contrat individuel de travail. Ils auraient beaucoup à y apprendre sur le thème de la rédaction des actes juridiques.

Ce livre d'accès facile devrait faire partie de la bibliothèque des spécialistes et des praticiens du droit du travail. En même temps, on ne peut s'empêcher de noter qu'un véritable traité sur les rapports individuels du travail reste à écrire. Avis aux générations montantes.

Claude D'Aoust

Université de Montréal

The Re-Emergence of Small Enterprises. Industrial Restructuring in Industrialised Countries, edited by Werner Sengenberger, Gary W. Loveman and Michael J. Piore, Genève, International Institute for Labour Studies, 1990, 308 p., ISBN 92-9014-465-3

L'Institut international d'études sociales (BIT) publie un recueil de six monographies nationales ainsi qu'une synthèse de ces différentes études portant sur la réalité des petites et moyennes entreprises dans les pays industrialisés. Chaque monographie est rédigée par un chercheur du pays en question. Les pays retenus et les auteurs des monographies sont les suivants: la France, avec Jean-François Amadieu; la République fédérale d'Allemagne, avec Stephanie Weimer; I'Italie, avec Giacomo Becattini; le Japon, avec Kazutoshi Koshiro; le Royaume-Uni, avec David Marsden et finalement les ÉtatsUnis, avec Michael J. Piore. Le chapitre d'introduction, qui constitue en fait la synthèse des différentes monographies nationales, est rédigé par Gary Loveman et Werner Sengenberger.

L'intérêt de l'IIES pour cette question s'explique par le fait que la part de l'emploi dans les PME a augmenté partout depuis la fin des années 70 . Étant donné que les conditions de travail varient selon la taille des entreprises, il était normal que l'organisme chargé de l'établissement des normes internationales de travail se préoccupe de la question. Chaque monographie nationale essaie donc de répondre à une même série de questions, à savoir l'importance quantitative du

3 Voir le Dictionnaire de droit privé et Lexiques bilingues, Centre de recherche de droit privé \& comparé du Québec, deuxième édition, Cowansville (Québec), Les Éditions Yvon Blais, 1991, p. 120. 